\title{
Clinical factors associated with death in 3044 COVID-19 patients managed in internal medicine wards in Italy: comment
}

\author{
Alessandra Bandera ${ }^{1,2} \cdot$ Alessandro Nobili $^{3} \cdot$ Mauro Tettamanti $^{3} \cdot$ Sergio Harari ${ }^{4} \cdot$ Silvano Bosari $^{5}$. \\ Pier Mannuccio Mannucci ${ }^{6}$ - $\cdot$ COVID-19 Network Working Group
}

Received: 11 June 2021 / Accepted: 14 June 2021 / Published online: 18 August 2021

(c) Società Italiana di Medicina Interna (SIMI) 2021

\section{Dear Editor,}

Recently in this journal Corradini et al. [1] reported the results of a nationwide registry of 3044 patients (mean age $67 \pm 15$ years) hospitalized in Italian internal medicine wards during the time period spanning from February 2 to May 8, 2020, when the country was the first in Europe and the second in the world after China to be dramatically hit by the infection with the coronavirus SARS-CoV2 and the dire clinical manifestations of COVID-19. The main results obtained from the registry data collected in 41 medical wards across the country showed that 351 infected patients died during their hospital stay in the medical wards, with an additional 310 cases who died after transferral to intensive care units (ICU), with a total in-hospital mortality rate of $21.7 \%(n=661)$.

In the context of these findings, Corradini et al. [1] chose to evaluate which patient features recorded at the time of

The members of "COVID-19 Network Working Group" are presented in the Acknowledgements section.

Pier Mannuccio Mannucci

piermannuccio.mannucci@policlinico.mi.it

1 Infectious Diseases Unit, Fondazione IRCCS Ca' Granda Ospedale Maggiore Policlinico, Milan, Italy

2 Department of Pathophysiology and Transplantation, University of Milan, Milan, Italy

3 Department of Neuroscience, Istituto di Ricerche Farmacologiche Mario Negri IRCCS, Via Mario Negri 2, 20156 Milan, Italy

4 Division of Pulmonary and Critical Care Medicine, San Giuseppe Hospital MultiMedica IRCCS, Milan, Italy

5 Scientific Direction, Fondazione IRCCS Ca' Granda Ospedale Maggiore Policlinico, Milan, Italy

6 Fondazione IRCCS Ca' Granda Ospedale Maggiore Policlinico, Angelo Bianchi Bonomi Hemophilia and Thrombosis Center, Via Pace 9, 20122 Milano, Italy admission were positively or negatively associated with mortality. First and importantly, age was independently associated with in-hospital death (OR 2.46). The authors also evaluated the association of death with the main symptoms on admission and found that the presence of productive cough was positively associated, this symptom being a plausible proxy of bacterial superinfection, whereas fever was negatively associated with death, perhaps a proxy of a useful inflammation response. Then the authors evaluated the association with deaths of a number of pre-existing or current comorbidities recorded at admission. The strongest signals of positive associations were for dementia (OR 1.90), chronic heart failure (OR 1.58), atrial fibrillation (OR 1.51), chronic obstructive pulmonary disease (OR 1.23) and chronic renal failure (OR 1.30). Other variables recorded at admission with a positive association with mortality were the number of pre-existing comorbidities (OR 1.36) and domiciliary polypharmacy (OR 1.20) [1], in broad agreement with a previous nationwide observational study [2].

With these results as background, we elected to compare them with those obtained in the frame of another registry conducted in the same period of the first burst of SARS$\mathrm{CoV}-2$ infection under the initiative and coordination of a research hospital that acted as a hub for COVID-19 in the metropolitan area of Milan, the capital city of the Northern Italian Region Lombardy [3]. This most populated Italian region (approximately 10 million, $16 \%$ of the country population) had the peculiar feature of being the first to be heavily hit from the pandemic from February to May 2020. The registry was also promoted by the Mario Negri Institute of Pharmacological Research that, besides preparing the protocol and case report form together with the hub hospital, did the data analysis. A few hospitals, mainly from Lombardy but also Veneto, Abruzzo and Emilia Romagna, chose to join the registry, but $80 \%$ of patients were enrolled in the two research and teaching hospitals in Milan, i.e., IRCCS 
Maggiore Policlinico (who contributed for $61 \%$ of the study population) and San Giuseppe Multimedica (15\%).

At variance with the patients reported by Corradini et al. [1] admitted upfront to medical wards, 1018 patients with COVID-19 (mean age $65 \pm 16$ ) enrolled in this registry were first screened at the emergency room and found to be positive for SARS-CoV2 RNA by RT-PCR. Then those who, owing to their moderately severe pulmonary involvement, did not need admission to intensive care units were preferentially admitted to pneumology wards, where continuous positive airway pressure (CPAP) non-invasive ventilation (NIV) or high flow-nasal cannula (HFNC) was initiated [4]. When lung involvement was relatively mild patients were preferentially admitted to infectious disease and internal medicine wards, where they were mainly handled by means of conventional oxygen supplementation (i.e., nasal cannula, oral nasal face mask, ventimask, reservoir) and more seldom with CPAP/NIV. This triage strategy was quite effective, because only a minority of patients admitted to these hospital wards needed transfer to intensive care units (3.1\%). All admitted patients were evaluated and data collected until discharge or death.

To offer a comparison with the results obtained by Corradini et al. [1] in internal medicine wards, we chose like them to compare the patients who died $(n=220)$ with those who survived $(n=798)$ (mortality rate $21.6 \%)$, by means of logistic regression analysis adjusted for age and sex and multivariable logistic regression adjusted also for the comorbidities ascertained at admission, in order to assess the association between a number of variables and death. Table 1 reports the results, with particular but not exclusive emphasis on the variables analyzed and reported by Corradini et al. [1]. Our findings in the frame of the first and most heavily affected Italian region are largely confirmatory of those of the nationwide SIMI cohort. On statistical analysis fully adjusted for all comorbidities, there was a positive association of age with in-hospital mortality (OR 1.07, CI 1.05-1.09), particularly strong for the quintiles above 75 years [OR for $75-84$ years, 7.78 (2.26-26.8); for 85 or more OR 11.8 (3.27-42.5)]. We also found that respiratory and heart rate measured at hospital entry were positively associated with mortality, as well as body temperature. Systolic and diastolic blood pressure were negatively associated, perhaps as a proxy of a greater cardiovascular reserve. We also confirm the findings of Corradini et al. that the number of comorbidities and chronic polypharmacy were associated with mortality, and that dementia and chronic heart failure were the pre-existing comorbidities recorded at admission more strongly associated. This was also true but with lower strength for cancer and diabetes, with a definite trend of positive association for a history of myocardial infarction and atrial fibrillation but only in the frame of the univariate analysis. At variance with Corradini et al. [1], COPD was not associated with the mortality risk but this difference may
Table 1 Variables recorded at admission and their association with in-hospital mortality

\begin{tabular}{llrrr}
\hline Variable & OR $(95 \% \mathrm{CI})^{*}$ & $P$ value & OR $(95 \% \text { CI })^{* *}$ & $P$ value \\
\hline Age & $1.09(1.07-1.10)$ & $<0.001$ & $1.07(1.05-1.09)$ & $<0.001$ \\
Body temperature & $1.16(0.95-1.41)$ & 0.154 & $1.30(1.03-1.64)$ & 0.026 \\
Heart rate & $1.02(1.01-1.04)$ & $<0.001$ & $1.02(1.01-1.04)$ & 0.001 \\
Respiratory rate & $1.13(1.09-1.16)$ & $<0.001$ & $1.13(1.09-1.17)$ & $<0.001$ \\
Systolic BP & $0.98(0.97-0.99)$ & $<0.001$ & $0.99(0.98-0.99)$ & 0.008 \\
Diastolic BP & $0.98(0.96-0.99)$ & 0.006 & $0.99(0.97-1.00)$ & 0.096 \\
Number of comorbidities & $1.44(1.31-1.59)$ & $<0.001$ & $1.80(1.15-2.80)$ & 0.010 \\
Number of drugs & $1.08(1.04-1.12)$ & $<0.001$ & $1.07(1.02-1.12)$ & 0.002 \\
Dementia & $3.03(1.75-5.24)$ & $<0.001$ & $4.60(2.48-8.52)$ & 0.001 \\
Chronic heart failure & $3.38(1.89-6.05)$ & $<0.001$ & $3.51(1.73-7.16)$ & $<0.001$ \\
Cancer & $3.34(1.93-5.78)$ & $<0.001$ & $3.43(1.87-6.29)$ & $<0.001$ \\
Diabetes & $2.04(1.38-3.00)$ & $<0.001$ & $1.63(1.04-2.56)$ & 0.035 \\
COPD & $1.58(0.97-2.56)$ & 0.064 & $0.89(0.48-1.63)$ & 0.700 \\
Asthma & $0.42(0.14-1.29)$ & 0.130 & $0.48(0.12-1.86)$ & 0.289 \\
Previous myocardial infarction & $2.20(1.39-3.49)$ & 0.001 & $1.69(0.99-2.88)$ & 0.054 \\
Previous stroke/TIA & $1.72(0.99-2.98)$ & 0.054 & $1.15(0.60-2.19)$ & 0.680 \\
Atrial fibrillation & $1.64(1.02-2.63)$ & 0.042 & $1.62(0.92-2.84)$ & 0.094 \\
Malnutrition & $9.35(3.04-28.76)$ & $<0.001$ & $17.20(3.19-92.65)$ & 0.001 \\
\hline
\end{tabular}

Univariate analysis (adjusted for sex and age) (left column), multivariate analysis adjusted also for comorbidities (right column)

*Age and sex adjusted

**Adjusted for age, sex and all comorbidities 
be explained by the size of our cohort that was one third smaller than that based on the whole country. Regarding oxygen supplementation, similarly to the findings from the SIMI-COVID-19 cohort, $69.6 \%$ of patients enrolled in our registry was treated with oxygen support during hospitalization and $20.5 \%$ received non-invasive ventilation (CPAP, NIV or HFNC). At multivariable analysis, when compared to subjects without oxygen support, patients who needed non-invasive ventilation had a significantly higher risk of in-hospital mortality (OR 4.31, 2.69-6.89), while the need for low-flow oxygen support was not associated with the risk of death (OR 0.84, 0.57-1.25).

All in all, the combined perusal of registry data obtained from these Italian cohorts is justified because, even though the criteria chosen for admission to hospital wards were different, age and total mortality rates were quasi identical in patients hospitalized during the first bout of the SARSCoV-2 pandemic. Both data broadly confirm the primary role of aging and pre-existing chronic diseases in causing the similarly high mortality rate observed in both cohorts. Like Corradini et al. [1], we chose to evaluate the potency of the association with death of variables recorded at admission because a more focused therapeutic approach on the basis of these symptoms and comorbidities may help to better plan and tailor the therapeutic strategies, at a time when specific antiviral agents were not available for COVID-19.

Acknowledgements Co-authors of the "COVID-19 NETWORK" WORKING GROUP are: Fondazione IRCCS Ca' Granda Ospedale Maggiore Policlinico. Scientific Direction: Silvano Bosari, Luigia Scudeller, Giuliana Fusetti, Laura Rusconi, Silvia Dell'Orto; Department of Transfusion Medicine and Hematology (Biobank): Daniele Prati, Luca Valenti, Silvia Giovannelli, Maria Manunta, Giuseppe Lamorte, Francesca Ferrari; Infectious Diseases Unit: Andrea Gori, Alessandra Bandera, Antonio Muscatello, Davide Mangioni, Laura Alagna, Giorgio Bozzi, Andrea Lombardi, Riccardo Ungaro, Giuseppe Ancona, Gianluca Zuglian, Matteo Bolis, Nathalie Iannotti, Serena Ludovisi, Agnese Comelli, Giulia Renisi, Simona Biscarini Valeria Castelli, Emanuele Palomba, Marco Fava, Valeria Fortina, Carlo Alberto Peri, Paola Saltini, Giulia Viero, Teresa Itri, Valentina Ferroni, Valeria Pastore, Roberta Massafra, Arianna Liparoti, Toussaint Muheberimana; Alessandro Giommi, Rosaria Bianco, Rafaela Montalvao De Azevedo, Grazia Eliana Chitani; Angelo Bianchi Bonomi Hemophilia and Thrombosis Center and Fondazione Luigi Villa: Flora Peyvandi, Roberta Gualtierotti, Barbara Ferrari, Raffaella Rossio, Nadia Boasi, Erica Pagliaro, Costanza Massimo, Michele De Caro; UOC Internal Medicine, Immunology and Allergology: Nicola Montano, Barbara Vigone, Chiara Bellocchi, Angelica Carandina, Elisa Fiorelli, Valerie Melli, Eleonora Tobaldini; Respiratory Unit and Cystic Fibrosis Adult Center: Francesco Blasi, Stefano Aliberti, Maura Spotti, Leonardo Terranova, Sofia Misuraca, Alice D’Adda, Silvia Della Fiore, Marta Di Pasquale, Marco Mantero Martina Contarini, Margherita Ori, Letizia Morlacchi, Valeria Rossetti, Andrea Gramegna, Maria Pappalettera, Mirta Cavallini, Agata Buscemi; Cardiology Unit: Marco Vicenzi, Irena Rota. Emergency Unit: Giorgio Costantino, Monica Solbiati, Ludovico Furlan, Marta Mancarella, Giulia Colombo, Giorgio Colombo, Alice Fanin, Mariele Passarella; Acute Internal Medicine: Valter Monzani, Ciro
Canetta, Angelo Rovellini, Laura Barbetta, Filippo Billi, Christian Folli, Silvia Accordino; Rare Diseases Center: Diletta Maira, Cinzia Maria Hu, Irene Motta, Natalia Scaramellini; General Medicine and Metabolic Diseases: Anna Ludovica Fracanzani, Rosa Lombardi, Annalisa Cespiati; Geriatric Unit: Matteo Cesari, Tiziano Lucchi, Marco Proietti, Laura Calcaterra, Clara Mandelli, Carlotta Coppola, Arturo Cerizza. Intensive Care Unit: Antonio Maria Pesenti, Giacomo Grasselli, Alessandro Galazzi. Istituto di Ricerche Farmacologiche Mario Negri IRCCS: Alessandro Nobili, Mauro Tettamanti, Igor Monti, Alessia Antonella Galbussera. Policlinico G.B. Rossi, Verona: UOC di Medicina d'Urgenza: Ernesto Crisafulli, Domenico Girelli, Alessio Maroccia, Daniele Gabbiani, Fabiana Busti, Alice Vianello, Marta Biondan, Filippo Sartori. Ospedale San Gerardo, ASST Monza: UOC Pneumologia: Paola Faverio, Alberto Pesci, Stefano Zucchetti. Malattie Infettive: Paolo Bonfanti, Marianna Rossi, Ilaria Beretta, Anna Spolti. San Giuseppe Hospital MultiMedica IRCCS and community health, Università degli Studi di Milano: UOC Pneumologia: Sergio Harari. Unità di Pneumologia e terapia Semi-intensiva respiratoria, Servizio di Fisiopatologia Respiratoria ed Emodinamica Polmonare: Davide Elia. Unità di Pneumologia e terapia Semi-intensiva respiratoria, Servizio di Fisiopatologia Respiratoria ed Emodinamica Polmonare: Roberto Cassandro, Antonella Camninati. Ospedale Clinicizzato "SS. Annunziata": Clinica Medica: Francesco Cipollone, Maria Teresa Guagnano, Damiano D’Ardes, Ilaria Rossi, Francesca Vezzani. ICS Maugeri Tradate, Universita' Insubria: Pneumologia Riabilitativa: Antonio Spanevello, Francesca Cherubino, Dina Visca. Azienda Ospedaliera Universitaria di Ferrara e Dipartimento di Medicina Traslazionale Università di Ferrara: UO Pneumologia: Marco Contoli, Alberto Papi, Luca Morandi, Nicholas Battistini. Clinica Polispecialistica San Carlo: UO Medicina Interna: Guido Luigi Moreo, Pasqualina Iannuzzi. UO Oncologia: Daniele Fumagalli. UO Chirurgia Generale: Sara Leone.

Funding The authors received no financial support for this study and have no conflict of interest.

\section{Declarations}

Conflict of interest The authors declare that they have no conflict of interest.

Statement of human and animal rights This article does not contain any studies with humans and participants or animals performed by any of the authors.

Informed consent For this type of study, formal consent is not required.

\section{References}

1. Corradini E, Ventura P, Ageno W et al (2021) Clinical factors associated with death in 3044 COVID-19 patients managed in internal medicine wards in Italy: results from the SIMI-COVID-19 study of the Italian Society of Internal Medicine (SIMI). Intern Emerg Med. https://doi.org/10.1007/ s11739-021-02742-8

2. Polverino F, Stern DA, Ruocco G et al (2020) Comorbidities, cardiovascular therapies, and COVID-19 mortality: a nationwide, Italian observational study (ItaliCO). Front Cardiovasc Med. https://doi.org/10.3389/fcvm.2020.585866

3. Bandera A, Aliberti S, Gualtierotti R et al (2020) COVID19 Network: the response of an Italian Reference Institute to 
research challenges about a new pandemia. Clin Microbiol Infect 26:1576-1578

4. Vitacca M, Nava S, Santus P, Harari S (2020) Early consensus management for non-ICU acute respiratory failure SARSCoV-2 emergency in Italy: from ward to trenches. Eur Respir J $55: 2000632$
Publisher's Note Springer Nature remains neutral with regard to jurisdictional claims in published maps and institutional affiliations. 